\title{
Composite Hermite-Birkhoff Quadrature Formulas of Gaussian Type*
}

\author{
By Nira Dyn
}

\begin{abstract}
We show how to combine incidence matrices, which admit Hermite-Birkhoff quadrature formulas of Gaussian type for any positive measure, in such a way that the resulting matrix also admits Gaussian type quadratures for any positive measure. Moreover, the uniqueness property and the extremal property of the formulas corresponding to the submatrices are transferred to the formula admitted by the composed matrix.
\end{abstract}

1. Introduction. Recently results concerning the existence of quadrature formulas of Gaussian type related to Hermite-Birkhoff interpolation problems have been obtained by several authors [4], [1], [2], [3].

Given the incidence matrix $E=\left\{e_{i j}\right\}_{i=0, j=0}^{m+1 n-1}$ with entries consisting of zeros and at most $n$ ones, a Hermite-Birkhoff quadrature formula (HB-QF) is defined as a formula of the form

$$
\int_{a}^{b} p d \sigma=\sum_{e_{i j}=1} a_{i j} p^{(j)}\left(x_{i}\right), \quad p \in \Pi_{n-1} .
$$

The formula (1.1) is called Hermite-Birkhoff Gaussian quadrature formula (HBGQF) [1], if the number of parameters in (1.1) equals the dimension of $\Pi_{n-1}$ (the space of polynomials of degree $\leqslant n-1)$, namely if

$$
n=\sum_{i=0}^{m+1} \sum_{j=0}^{n-1} e_{i j}+m
$$

In (1.1) $d \sigma$ is a nonnegative measure supported on more than $m$ points in $(a, b)$, $\left(x_{0}, \ldots, x_{m+1}\right) \in S^{m}$ where

$$
S^{m}=\left\{Y=\left(y_{0}, \ldots, y_{m+1}\right) \mid a=y_{0}<y_{1}<\cdots<y_{m}<y_{m+1}=b\right\}
$$

and

$$
\sum_{j=0}^{n-1} e_{i j}>0, \quad 1 \leqslant i \leqslant m, \quad \sum_{j=0}^{n-1} e_{i j} \geqslant 0, \quad i=0 \text { or } m+1
$$

Received July 1, 1982; revised December 7, 1983.

1980 Mathematics Subject Classification. Primary 41A05, 41A55.

Key words and phrases. Gaussian quadrature formulas, Hermite-Birkhoff interpolation.

*Partially done at the Mathematics Research Center, University of Wisconsin-Madison. Sponsored by the United States Army under Contract No. DAAG29-80-C-0041.

(C)1984 American Mathematical Society $0025-5718 / 84 \$ 1.00+\$ .25$ per page 
This notion of HB-GQF extends the classical notion of Gaussian quadrature formulas (GQF):

$$
\int_{a}^{b} p d \sigma=\sum_{i=1}^{m} a_{i} p\left(x_{i}\right), \quad p \in \Pi_{2 m-1}
$$

where $a<x_{1}<\cdots<x_{m}<b$ and $a_{i}>0, i=1, \ldots, m$, and also the notion of the multiple nodes GQF [8], [7].

The problem of characterizing incidence matrices admitting HB-QF is posed in [4], and the following necessary condition on such matrices is proved:

Result A. Let $E=\left\{e_{i j}\right\}_{i=0, j=0}^{m+1 ~} n-1$ admit a quadrature formula exact for $\Pi_{n-1}$, for some nonnegative measure $d \sigma$ supported on more than $m$ points in $[a, b]$, and let $r$ be the minimal number of ones which must be added to $E$ to obtain a matrix $\tilde{E}=\left\{\tilde{e}_{i j}\right\}_{i=0, j=0}^{m+1 n-1}$ without odd sequences in rows $1, \ldots, m$. Then $\tilde{E}$ is a Pólya matrix (the number of ones in any first $l$ columns exceeds $l-1$ ), and

$$
n \leqslant \sum_{i=0}^{m+1} \sum_{j=0}^{n-1} e_{i j}+r .
$$

In [2] the existence of two classes of quadrature formulas satisfying (1.3) with equality, has been claimed. The first consists of HB-GQF related to incidence matrices with rows $1, \ldots, m$ consisting of odd Hermite sequences and even nonHermite sequences. The existence of this class of HB-GQF is proved in [1].

The present paper is concerned with the proof of the existence of the second class of composite HB-GQF, corresponding to incidence matrices which can be decomposed vertically into several submatrices, each admitting a HB-GQF with equality in (1.3). It is also proved here that such a formula is unique and/or has an extremal property in case each of the HB-GQF corresponding to the submatrices is unique and/or has an extremal property of the following type:

Definition 1. The HB-GQF (1.1) related to $E=\left\{e_{i j}\right\}_{i=0, j=0}^{m+1}$, with $e_{i, n-1}=0$, $1 \leqslant i \leqslant m$, has an extremal property, if for any $f$ satisfying $f^{(n)} \geqslant 0$ on $[a, b]$ :

$$
\min _{p \in P(E, f)}\left[(-1)^{\mu} \int_{a}^{b}(f-p) d \sigma\right]=(-1)^{\mu}\left[\int_{a}^{b} f d \sigma-\sum_{e_{i j}=1} a_{i j} f^{(j)}\left(x_{i}\right)\right]
$$

where $\mu=\sum_{j=0}^{n-1} e_{m+1, j}$ and

$$
P(E, f)=\left\{p \mid p \in \Pi_{n-1}, p^{(j)}\left(y_{i}\right)=f^{(j)}\left(y_{i}\right), \tilde{e}_{i j}=1, Y \in S^{m}\right\},
$$

with $\tilde{E}=\left\{\tilde{e}_{i j}\right\}$ the matrix of Result A obtained from $E$ by addition of ones at the end of odd sequences.

The existence of another interesting class of HB-GQF has been established in [3]. These formulas are related to incidence matrices of pyramidal type:

(a) Each interior row, $i$, contains one sequence only which is of odd length $l_{i}$ starting at column $k_{i}$.

(b) There exist $1 \leqslant I \leqslant J \leqslant m$ such that $k_{i}+l_{i} \geqslant k_{i-1} \geqslant k_{i}, i=2, \ldots, I, k_{i}=0$, $i=I, \ldots, J, k_{i}+l_{i} \geqslant k_{i+1} \geqslant k_{i}, i=J, \ldots, m-1$.

(c) The two rows $i=0, i=m+1$ have nonzero entries in arbitrary positions $k \geqslant k_{1}$ and $k \geqslant k_{m}$ respectively.

The proof of the existence in [3] is based on the extremal property of Definition 1, characterizing several other classes of HB-GQF, in particular the classical GQF and 
the multiple nodes GQF [8], [7]. This class of matrices, as well as the class in [1], can be used in constructing new composite HB-GQF of the type discussed in Section 2.

2. Existence and Uniqueness. Given a regular incidence matrix $E=\left\{e_{i j}\right\}_{i=0, j=0}^{m+1} n-1$ with $n$ ones, and a set of points $X \in S^{m}$, denote by $p(E, X, f)(x)$ the interpolating polynomial from $\Pi_{n-1}$ to $f \in C^{n-1}[a, b]$ at the data $(E, X)$,

$$
p(E, X, f)^{(j)}\left(x_{i}\right)=f^{(j)}\left(x_{i}\right), \quad e_{i j}=1 .
$$

( $E$ is regular if (2.1) is solvable for any $X \in S^{m}$ and data $f^{(j)}\left(x_{i}\right), e_{i j}=1$.) The error in the interpolation prescribed by $(E, X)$ is given in terms of the Peano kernel $K(t, x) \equiv K(t, x \mid E, X)$, by [5, Theorem 7.4]:

$$
f(x)-p(E, X, f)(x)=\int_{a}^{b} K(t, x \mid E, X) f^{(n)}(t) d t .
$$

It is well known [5, Theorem 7.7] that if $E$ is a Pólya matrix such that all its non-Hermite sequences in the interior rows (rows $1, \ldots, m$ ) are even, then $E$ is regular and the sign of $K(t, x)$ is constant in $t$ for any fixed $x \in[a, b]$. For such matrices, with even Hermite sequences in the interior rows as well, the sign of $K(t, x)$, as a function of $x$, can also be determined, in view of the following result.

Result B [3, Lemma 2.2]. Let $E$ be a Pólya matrix with even sequences only in its interior rows, and let $f=x^{n} / n$ ! Then

$$
(-1)^{\mu}[f-P(E, X, f)] \geqslant 0 \quad \text { on }[a, b], \quad \mu=\sum_{j=0}^{n-1} e_{m+1, j} \geqslant 0 .
$$

Inserting $f=x^{n} / n$ ! into (2.2), we thus conclude:

COROllary 1. Let E be a Pólya matrix with even sequences only in its interior rows, and let $\mu=\sum_{j=0}^{n-1} e_{m+1, j}$. Then

$$
(-1)^{\mu} K(t, x \mid E, X) \geqslant 0, \quad(t, x) \in[a, b]^{2} .
$$

With this preliminary result, the existence of a composite HB-QF, corresponding to a vertically decomposable matrix, can be obtained.

Definition 2. Let $E=\left\{e_{i j}\right\}_{i=0, j=0}^{m+1}$ be an incidence matrix which can be vertically decomposed into $l$ submatrices, each consisting of $n_{i}$ columns and $m_{i}$ nonzero interior rows respectively,

$$
E=E\left(n_{1}\right) \oplus E\left(n_{2}\right) \oplus \cdots \oplus E\left(n_{l}\right), \quad \sum_{i=1}^{l} n_{i}=n,
$$

such that $\sum_{i=1}^{l} m_{i}=m$ (each interior row is a nonzero row of one submatrix only).

For each $1 \leqslant i \leqslant l$, let $Y^{(i)}$ denote the ordered subset of nodes of $Y=$ $\left(y_{0}, \ldots, y_{m+1}\right)$ corresponding to the nonzero rows of $E\left(n_{i}\right)$.

A composite HB-QF (CHB-QF) related to $E$ is defined as a quadrature formula of the form (1.1), with $X=\left(x_{0}, \ldots, x_{m+1}\right) \in \Omega^{m}$, where

$$
\Omega^{m}=\left\{Y=\left(y_{0}, \ldots, y_{m+1}\right) \mid Y^{(i)} \in S^{m_{i}}, 1 \leqslant i \leqslant l\right\} .
$$

A CHB-QF is Gaussian (CHB-GQF), if it satisfies (1.2).

Remark 1. Let $m^{*}$ denote the number of distinct nodes of a CHB-GQF. If $m^{*}<m$ then $n>\sum_{i=0}^{m+1} \sum_{j=0}^{n-1} e_{i j}+m^{*}$. Thus the number of monomials for which 
the CHB-GQF is exact exceeds the number of parameters in the quadrature formula (coefficients and nodes), in case of equality between nodes corresponding to nonzero interior rows of different submatrices.

The existence of CHB-GQF and their properties are stated and proved for the case $l=2$. The extension to the case $l>2$ is straightforward, as will become clear from the proofs.

THEOREM 1. Let $E$ satisfy the conditions of Definition 2 with $l=2$. If each $E\left(n_{i}\right)$, $i=1,2$, admits a HB-QF exact for $\Pi_{n_{i}-1}$ for any positive measure, and satisfies Result A with equality in (1.3), then $E$ admits a CHB-QF exact for $\Pi_{n-1}$ for any positive measure.

If, moreover, the $H B-Q F$ admitted by $E\left(n_{i}\right), i=1,2$, are Gaussian (satisfy (1.2)), then $E$ admits a CHB-GQF (satisfying (1.2)).

Proof. Let $\tilde{E}=\tilde{E}\left(n_{1}\right) \oplus \tilde{E}\left(n_{2}\right)$ where $\tilde{E}\left(n_{i}\right)$ is related to $E\left(n_{i}\right)$ by Result A. Let $Y \in S^{m_{1}}$ be the nodes of the HB-QF for $E\left(n_{1}\right)$ and the measure $d \sigma$, and let $Z \in S^{m_{2}}$ be the nodes of the HB-QF for $E\left(n_{2}\right)$ and the measure $\omega(t) d t$ with

$$
\omega(t)=\int_{a}^{b} d \sigma(x) K\left(t, x \mid \tilde{E}\left(n_{1}\right), Y\right) .
$$

That $\omega(t)$ is of constant sign follows from Corollary 1 .

Consider now the set of fundamental polynomials for interpolation at $(\tilde{E}, X)$ with $X \in \Omega^{m}, X^{(1)}=Y, X^{(2)}=Z$ :

$$
q_{i j}^{(k)}\left(x_{\nu}\right)=\delta_{i \nu} \delta_{j k}, \quad \tilde{e}_{k \nu}=1 .
$$

Then

$$
p(\tilde{E}, X, f)(x)=\sum_{\tilde{e}_{i j}=1} f^{(j)}\left(x_{i}\right) q_{i j}(x)
$$

and for any $p \in \Pi_{n-1}$,

$$
\begin{aligned}
p(x) & =\sum_{\tilde{e}_{i j}=1} p^{(j)}\left(x_{i}\right) q_{i j}(x), \\
\int_{a}^{b} p(x) d \sigma(x) & =\sum_{\tilde{e}_{i j}=1} p^{(j)}\left(x_{i}\right) \int_{a}^{b} q_{i j}(x) \dot{d} \sigma(x) .
\end{aligned}
$$

Now $\left\{q_{i j} \mid \tilde{e}_{i j}=1, j<n_{1}\right\}$ are the fundamental polynomials for $\Pi_{n_{1}-1}$, since their $n_{1}$ th derivatives all equal to $p\left(\tilde{E}\left(n_{2}\right), Z, 0\right) \equiv 0$. Using the HB-QF with nodes $Y \in S^{m_{1}}$ and coefficients $\left\{a_{i j} \mid e_{i j}=1, j<n_{1}\right\}$ admitted by $E\left(n_{1}\right)$, which is exact for $\Pi_{n_{1}-1}$, we obtain

$$
\begin{aligned}
\int_{a}^{b} q_{i j}(x) d \sigma(x) & =\sum_{\substack{e_{k \nu}=1 \\
\nu<n_{1}}} a_{k \nu} q_{i j}^{(\nu)}\left(y_{k}\right) \\
& =0 \text { if } e_{i j}=0 \text { and } \tilde{e}_{i j}=1,0 \leqslant j<n_{1} .
\end{aligned}
$$

Consider next the set $\left\{q_{i j} \mid \tilde{e}_{i j}=1, n_{1} \leqslant j<n\right\}$, consisting of $n_{2}$ polynomials in $\Pi_{n-1}$ vanishing on $\left(\tilde{E}\left(n_{1}\right), Y\right)$. By $(2.2)$, each of these polynomials can be represented as

$$
q_{i j}(x)=\int_{a}^{b} q_{i j}^{\left(n_{1}\right)}(t) K\left(t, x \mid \tilde{E}\left(n_{1}\right), Y\right) d t .
$$


Hence

$$
\begin{aligned}
\int_{a}^{b} q_{i j}(x) d \sigma(x) & =\int_{a}^{b} d t q_{i j}^{\left(n_{1}\right)}(t) \int_{a}^{b} K\left(t, x \mid \tilde{E}\left(n_{1}\right), Y\right) d \sigma(x) \\
& =\int_{a}^{b} q_{i j}^{\left(n_{1}\right)}(t) \omega(t) d t .
\end{aligned}
$$

But $q_{i j}^{\left(n_{1}\right)} \in \Pi_{n_{2}-1}$ for $e_{i j}=1, n_{1} \leqslant j<n$, and therefore the application of the HB-QF admitted by $E\left(n_{2}\right)$ for the measure $\omega(t) d t$, with nodes $Z \in S^{m_{2}}$ and coefficients $\left\{b_{i j} \mid e_{i, j+n_{1}}=1,0 \leqslant j<n_{2}\right\}$, yields

$$
\begin{aligned}
\int_{a}^{b} q_{i j}^{\left(n_{1}\right)} \omega(t) d t= & \sum_{\substack{e_{k \nu}=1 \\
n_{1} \leqslant \nu<n}} b_{k, \nu-n_{1}} q_{i j}^{(\nu)}\left(z_{k}\right)=0 \\
& \text { if } e_{i j}=0 \text { and } \tilde{e}_{i j}=1, n_{1} \leqslant j<n .
\end{aligned}
$$

Combining (2.11), (2.12), (2.14) and (2.15) we conclude that

$$
\int_{a}^{b} p d \sigma=\sum_{e_{i j}=1}\left[\int_{a}^{b} q_{i j}(x) d \sigma(x)\right] p^{(j)}\left(x_{i}\right), \quad p \in \Pi_{n-1} .
$$

Now if both HB-QF admitted by $E\left(n_{i}\right), i=1,2$, are Gaussian then

$$
n_{1}=\sum_{i=0}^{m+1} \sum_{j=0}^{n_{1}-1} e_{i j}+m_{1}, \quad n_{2}=\sum_{i=0}^{m+1} \sum_{j=n_{1}}^{n_{1}+n_{2}-1} e_{i j}+m_{2} \text {. }
$$

These together with the assumptions on $E: n=n_{1}+n_{2}, m=m_{1}+m_{2}$, yield

$$
n=\sum_{i=0}^{m+1} \sum_{j=0}^{n-1} e_{i j}+m \text {. }
$$

Using the same arguments it is easy to show that

THEOREM 2. Under the conditions of Theorem $1, E$ admits a CHB-QF at $X \in \Omega^{m}$ for the positive measure $d \sigma$, if and only if each $E\left(n_{i}\right), i=1,2$, admits a HB-QF at $X^{(i)} \in S^{m_{i}}$ for the positive measure $d \sigma_{i}$, where

$$
d \sigma_{i}=\left\{\begin{array}{l}
d \sigma, \quad i=1 \\
\omega_{2}(t) d t \equiv\left|\int_{a}^{b} K\left(t, x \mid \tilde{E}\left(n_{1}\right), X^{(1)}\right) d \sigma(x)\right| d t, \quad i=2
\end{array}\right.
$$

Remark 2 . In Theorem $2, \tilde{E}\left(n_{1}\right)$ is not necessarily uniquely determined. Nevertheless, it follows from the proof of Theorem 1 that if $E$ admits a CHB-QF at $X \in \Omega^{m}$ for the positive measure $d \sigma$, then for any measure $\omega_{2}(t) d t$ of the form (2.17), independently of the choice of $\tilde{E}\left(n_{1}\right), E\left(n_{2}\right)$ admits a HB-QF with $X^{(2)}$ as its set of nodes.

A direct conclusion from this remark and Theorems 1,2 is

COROLlaRY 2. Under the conditions of Theorem 1, the CHB-GQF admitted by $E$ at $X \in \Omega^{m}$ for the positive measure $d \sigma$ is unique, if and only if $E\left(n_{1}\right)$ admits a unique $H B-G Q F$ at $X^{(1)} \in S^{m_{1}}$ for do, and $E\left(n_{2}\right)$ admits a unique HB-GQF for at least one measure $d \sigma_{2}$ of the form (2.17).

Remark 3. The uniqueness result in Corollary 2 and the extremal property in Section 3 are formulated and proved for CHB-GQF. The proofs are based on the 
same results valid for the HB-GQF admitted by the submatrices, and are independent of property (1.2). Therefore similar results hold for CHB-QF, but in fact are empty, since the uniqueness and extremal property are only valid for certain types of HB-GQF; cf. [7], [8], [6], [3].

3. The Extremal Property of a CHB-GQF. A property of the HB-GQF admitted by $E\left(n_{i}\right), i=1,2$, which is transferred to the CHB-GQF admitted by $E\left(n_{1}\right) \oplus E\left(n_{2}\right)$ is the extremal property of Definition 1 . Note that (1.4) can be formulated also as

$$
\min _{p \in P(E, f)}\left[(-1)^{\mu} \int_{a}^{b}(f-p) d \sigma\right]=(-1)^{\mu} \int_{a}^{b}[f-p(\tilde{E}, X, f)] d \sigma .
$$

THEOREM 3. Let $E$ satisfy the conditions of Theorem 1 , and let each $E\left(n_{i}\right), i=1,2$, admit a $H B-G Q F$ with the extremal property of Definition 1 for any positive measure. Then for any positive measure, $E$ admits a CHB-GQF with the extremal property of Definition 1 but with $\tilde{E}$ and $S^{m}$ in the definition of $P(E, f)$ replaced by $\tilde{E}=\tilde{E}\left(n_{1}\right) \oplus$ $\tilde{E}\left(n_{2}\right)$ and $\Omega^{m}$, respectively, where $\tilde{E}\left(n_{i}\right)$ is related to $E\left(n_{i}\right)$ as in Definition 1.

Proof. Given $f^{(n)} \geqslant 0$ on $[a, b]$ and arbitrary $U \in \Omega^{m}$, define $g_{1}=f-p(\tilde{E}, U, f)$. Since $g_{1}^{\left(n_{1}\right)}$ is the error in the interpolation of $f^{\left(n_{1}\right)}$ at $\left(\tilde{E}\left(n_{2}\right), U^{(2)}\right)$, relation (2.2) and Corollary 1 imply that

$$
\begin{aligned}
g_{1}^{\left(n_{1}\right)}(x) & =\int_{a}^{b} g_{1}^{\left(n_{1}+n_{2}\right)}(t) K\left(t, x \mid \tilde{E}\left(n_{2}\right), U^{(2)}\right) d t \\
& =(-1)^{\mu_{2}} \int_{a}^{b} f^{(n)}(t)\left|K\left(t, x \mid \tilde{E}\left(n_{2}\right), U^{(2)}\right)\right| d t
\end{aligned}
$$

with $\mu_{2}=\sum_{n_{1} \leqslant j<n} e_{m+1, j}$. Therefore

$$
(-1)^{\mu_{2}} g_{1}^{\left(n_{1}\right)}(x) \geqslant 0, \quad x \in[a, b] .
$$

Now $0 \in P\left(E\left(n_{1}\right),(-1)^{\mu_{2}} g_{1}\right)$, and hence by the extremal property of the HB-GQF admitted by $E\left(n_{1}\right)$ with nodes $Y$, for the positive measure $d \sigma$ :

$$
(-1)^{\mu_{1}} \int_{a}^{b}(-1)^{\mu_{2}} g_{1} d \sigma \geqslant(-1)^{\mu_{1}} \int_{a}^{b}(-1)^{\mu_{2}}\left[g_{1}-q_{1}\right] d \sigma
$$

with $\mu_{1}=\sum_{j=0}^{n_{1}-1} e_{m+1, j}$ and $q_{1}=p\left(\tilde{E}\left(n_{1}\right), Y, g_{1}\right) \in \Pi_{n_{1}-1}$. In view of the definition of $g_{1}$ and $q_{1},(3.2)$ becomes

$$
(-1)^{\mu} \int_{a}^{b}[f-p(\tilde{E}, U, f)] d \sigma \geqslant(-1)^{\mu} \int_{a}^{b}[f-p(\tilde{E}, V, f)] d \sigma
$$

with $V \in \Omega^{m}, V^{(1)}=Y, V^{(2)}=U^{(2)}$ and $\mu=\mu_{1}+\mu_{2}$. To complete the proof we use again Corollary 1 and conclude that

$$
\begin{aligned}
(-1)^{\mu} & \int_{a}^{b}[f-p(\tilde{E}, V, f)](x) d \sigma(x) \\
& =(-1)^{\mu} \int_{a}^{b} \int_{a}^{b}[f-p(\tilde{E}, V, f)]^{\left(n_{1}\right)}(t) K\left(t, x \mid \tilde{E}\left(n_{1}\right), Y\right) d t d \sigma(x) \\
& =(-1)^{\mu} \int_{a}^{b} \omega(t)[f-p(\tilde{E}, V, f)]^{\left(n_{1}\right)}(t) d t
\end{aligned}
$$

where $\omega(t)$ is defined by (2.7), and satisfies $(-1)^{\mu_{1}} \omega(t) \geqslant 0$ on $[a, b]$. Applying the extremal property of the HB-GQF, admitted by $E\left(n_{2}\right)$ for $(-1)^{\mu_{1}} \omega(t) d t$ at the 
nodes $Z$, we obtain for $g_{2}=f^{\left(n_{1}\right)}, p_{2}=p^{\left(n_{1}\right)}(\tilde{E}, V, f)$, satisfying $g_{2}^{\left(n_{2}\right)}=f^{(n)} \geqslant 0$, $p_{2} \in P\left(E(2), g_{2}\right)$, the inequality

$$
\begin{array}{r}
(-1)^{\mu_{2}} \int_{a}^{b}\left[(-1)^{\mu_{1}} \omega(t)\right]\left[f^{\left(n_{1}\right)}-p^{\left(n_{1}\right)}(\tilde{E}, V, f)\right] d t \\
\geqslant(-1)^{\mu_{2}} \int_{a}^{b}\left[(-1)^{\mu_{1}} \omega(t)\right]\left[f^{\left(n_{1}\right)}-q_{2}\right](t) d t,
\end{array}
$$

where $q_{2}=p\left(\tilde{E}\left(n_{2}\right), Z, f^{\left(n_{1}\right)}\right) \in \Pi_{n_{2}-1}$. Now for $X \in \Omega^{m}, X^{(1)}=Y, X^{(2)}=Z$, $[f-p(\tilde{E}, X, f)]^{\left(n_{1}\right)}=f^{\left(n_{1}\right)}-q_{2}$, and we finally derive from (3.3), (3.4), and (3.5) that

$$
\begin{aligned}
& (-1)^{\mu} \int_{a}^{b}[f-p(\tilde{E}, U, f)] d \sigma \geqslant(-1)^{\mu_{2}} \int_{a}^{b}[f-p(\tilde{E}, X, f)]^{\left(n_{1}\right)}(-1)^{\mu_{1}} \omega d t \\
& \quad=(-1)^{\mu} \int_{a}^{b}[f-p(\tilde{E}, X, f)] d \sigma
\end{aligned}
$$

indicating the optimal property of the HB-GQF admitted by $E$ at $X$.

The following two matrices are examples of matrices which admit HB-GQF in view of Theorem 1 and the results in [1] and [3]:

$$
\begin{aligned}
E_{1} & =\left(\begin{array}{llllll}
1 & 1 & 0 & 0 & 0 & 0 \\
1 & 0 & 1 & 1 & 0 & 0 \\
0 & 0 & 0 & 0 & 0 & 0 \\
0 & 0 & 0 & 0 & 0 & 0
\end{array}\right)\left(\begin{array}{llllll}
1 & 0 & 1 & 0 & 0 & 0 \\
0 & 0 & 0 & 0 & 0 & 0 \\
1 & 0 & 0 & 1 & 1 & 0 \\
0 & 0 & 0 & 0 & 0 & 0
\end{array}\right), \\
E_{2} & =\left(\begin{array}{llllllll}
0 & 1 & 0 & 0 & 0 & 0 & 0 & 0 \\
0 & 1 & 1 & 1 & 0 & 0 & 0 & 0 \\
0 & 0 & 0 & 0 & 0 & 0 & 0 & 0 \\
1 & 0 & 0 & 0 & 0 & 0 & 0 & 0 \\
0 & 0 & 1 & 0 & 0 & 0 & 0 & 0
\end{array}\right)\left(\begin{array}{lllll}
0 & 1 & 0 & 0 & 0 \\
0 & 0 & 0 & 0 & 0 \\
1 & 1 & 1 & 0 & 0 \\
0 & 0 & 0 & 0 & 0 \\
0 & 0 & 0 & 0 & 0
\end{array}\right)
\end{aligned}
$$

The matrix $E_{2}$ admits a HB-GQF with the extremal property of Theorem 3, in view of the results in [3], [6] concerning the two submatrices of $E_{2}$.

School of Mathematical Sciences

Tel-Aviv University

Tel-Aviv, Israel

1. N. Dyn, “On the existence of Hermite-Birkhoff quadrature formulas of Gaussian type," J. Approx. Theory, v. 31, 1981, pp. 22-32.

2. N. DYN, "Hermite-Birkhoff quadrature formulas of Gaussian type," in Approximation Theory III (E. W. Cheney, ed.), Academic Press, New York, 1980, pp. 371-376.

3. K. JETTER, "A new type of Gaussian quadrature formulas based on Birkhoff type data," SIAM J. Numer. Anal., v. 19, 1982, pp. 1081-1089.

4. G. G. LORENTZ \& S. D. RiEMENSCHNEIDER, "Birkhoff quadrature matrices," in Linear Spaces and Approximation Theory (P. L. Butzer and B. Sz. Nagy, eds.), ISNM, Vol. 40, Birkhäuser-Verlag, Basel, 1978, pp. 359-373.

5. G. G. Lorentz, K. Jetter \& S. D. Riemenschneider, Birkhoff Interpolation, Addison-Wesley, Reading, Mass., 1983.

6. C. A. Micchelli \& T. J. Rivlin, "Quadrature formulae and Hermite-Birkhoff interpolation," Adv". in Math., v. 11, 1973, pp. 93-112.

7. T. Popovicıu, "Asupra unei generalizări a formulei de integrare numerică a lui Gauss," Acad. R. P. Romine Fil. Iaşi. Stud. Cerc. Sti., v. 6, 1955, pp. 29-57. (Roumanian)

8. P. TURÁN, "On the theory of mechanical quadrature," Acta Sci. Math. (Szeged), v. 12, 1950, pp. $30-37$. 